\title{
Outcome in Patients with Isolated Moderate to Severe Traumatic Brain Injury
}

\author{
D. Jochems $\mathbb{D}^{1}{ }^{1}$ K. J. P. van Wessem, ${ }^{1}$ R. M. Houwert, ${ }^{1}$ H. B. Brouwers, ${ }^{2}$ J. W. Dankbaar, ${ }^{3}$ \\ M. A. van Es, ${ }^{4}$ M. Geurts, ${ }^{4}$ A. J. C. Slooter, ${ }^{5}$ and L. P. H. Leenen ${ }^{1}$ \\ ${ }^{1}$ Department of Trauma, University Medical Center Utrecht, Utrecht University, Heidelberglaan 100, \\ 3585 GA Utrecht, Netherlands \\ ${ }^{2}$ Department of Neurosurgery, University Medical Center Utrecht, Utrecht University, Heidelberglaan 100, \\ 3585 GA Utrecht, Netherlands \\ ${ }^{3}$ Department of Radiology, University Medical Center Utrecht, Utrecht University, Heidelberglaan 100, \\ 3585 GA Utrecht, Netherlands \\ ${ }^{4}$ Department of Neurology, University Medical Center Utrecht, Utrecht University, Heidelberglaan 100, \\ 3585 GA Utrecht, Netherlands \\ ${ }^{5}$ Department of Intensive Care, University Medical Center Utrecht, Utrecht University, Heidelberglaan 100, \\ 3585 GA Utrecht, Netherlands
}

Correspondence should be addressed to D. Jochems; d.jochems-3@umcutrecht.nl

Received 8 May 2018; Revised 16 July 2018; Accepted 14 August 2018; Published 23 September 2018

Academic Editor: Thomas J. Esposito

Copyright $\odot 2018$ D. Jochems et al. This is an open access article distributed under the Creative Commons Attribution License, which permits unrestricted use, distribution, and reproduction in any medium, provided the original work is properly cited.

Introduction. Traumatic brain injury (TBI) remains a major cause of death. Withdrawal of life-sustaining treatment (WLST) can be initiated if there is little anticipated chance of recovery to an acceptable quality of life. The aim of this study was firstly to investigate WLST rates in patients with moderate to severe isolated TBI and secondly to assess outcome data in the survivor group. Material and Methods. A retrospective cohort study was performed. Patients aged $\geq 18$ years with moderate or severe isolated TBI admitted to the ICU of a single academic hospital between 2011 and 2015 were included. Exclusion criteria were isolated spinal cord injury and referrals to and from other hospitals. Gathered data included demographics, mortality, cause of death, WLST, and Glasgow Outcome Scale (GOS) score after three months. Good functional outcome was defined as GOS $>3$. Results. Of 367 patients, 179 patients were included after applying inclusion and exclusion criteria. 55 died during admission (33\%), of whom $45(82 \%)$ after WLST. Patients undergoing WLST were older, had worse neurological performance at presentation, and had more radiological abnormalities than patients without WLST. The decision to withdraw life-sustaining treatment was made on the day of admission in $40 \%$ of patients. In $33 \%$ of these patients, this decision was made while the patient was in the Emergency Department. $71 \%$ of survivors had a good functional outcome after three months. No patient left hospital with an unresponsive wakefulness syndrome (UWS) or suffered from UWS after three months. One patient died within three months of discharge. Conclusion. In-hospital mortality in isolated brain injured patients was $33 \%$. The vast majority died after a decision to withdraw life-sustaining treatment. None of the patients were discharged with an unresponsive wakefulness syndrome.

\section{Introduction}

In 2010, over 56,000 deaths in the USA and 57,000 in the European Union were related to traumatic brain injury (TBI) [1-3]. Furthermore, TBI is the main cause of death in severely injured trauma patients, contributing to $30 \%$ of the deaths caused by trauma $[1,2,4]$. Interestingly, mortality rates differ greatly between level-I trauma centers across the world [4].

TBI not only causes mortality, but can also lead to severe functional impairment. Unresponsive wakefulness syndrome (UWS) is a dreaded outcome, in which the patient 
does not demonstrate any sign of consciousness [5]. Withdrawal of life-sustaining treatment (withdrawal of treatment; WLST) can be initiated when treatment is considered medically futile, in cases where there is negligible chance of recovery to an acceptable quality of life $[1,5,6]$.

The Ethicus study [7] investigated end-of-life practices in various ICUs across Europe. Differences in practices between these hospitals included a higher WLST rate in Northern and Central European countries, when compared to countries in Southern Europe. Furthermore, the length of ICU stay before the first treatment limiting decision was significantly shorter in Northern Europe than in the rest of the continent. Amongst patients with acute conditions, neurological disease was the most common motive for treatment limitations [7]. Moreover, a retrospective study in a Dutch ICU found that this was true for WLST as well [8]. However, few studies have published WLST rates, especially not in combination with neurological or functional outcome data.

Therefore, the aim of this study was to investigate WLST rates in patients with moderate to severe isolated TBI and to assess outcome data of the survivors.

\section{Material and Methods}

A local institutional review board (IRB) waiver was formally obtained.

2.1. Study Design and Study Population. A retrospective cohort study was conducted including all consecutive patients who sustained isolated moderate or severe traumatic brain injury and were admitted to ICU of the University Medical Center Utrecht (UMCU, a level-1 trauma center) between 2011 and 2015. Isolated moderate or severe brain injury was defined as an Abbreviated Injury Score head \& neck (AIShead) of more than three and no significant injury in other regions (defined as AIS of more than two). Patients under 18 years of age, patients with isolated spinal injury without TBI, and referrals to and from other hospitals were excluded. If first CT head showed only subdural and/or parenchymal hemorrhage, patients' records were checked and patients were excluded from analysis, if there was any doubt on whether the brain injury was the consequence or the cause of trauma.

Patients who passed away without WLST were only analyzed for cause of death. This decision was based on the hypothesis that this excluded group will be relatively small, and our main interest was in WLST.

2.2. Clinical Variables. Data were collected from medical records and the local trauma database. This database includes several baseline characteristics such as age, sex, ISS, and the AIS of the head region. The trauma mechanism was collected from the medical records. Collected variables included: the Glasgow Coma Scale (GCS) as assessed by the neurologist during the primary survey; pupillary light reflexes and corneal reflexes during primary survey; the need for sedation before arrival or during the stay in the
Emergency Department (ED), the concurrent use of a low molecular weight heparin (LMWH); and coumarin or a novel oral anticoagulant (NOAC).

The Charlson Comorbidity Index score was calculated for every patient. This is a widely used score for comorbidity, which comprises 22 comorbidities and each is assigned a weight, according to its impact on the prognosis of the patient $[9,10]$.

2.3. Imaging Variables. In each patient, a noncontrast CT of the head was acquired within 30 minutes after arrival to ED. An experienced neuroradiologist, blinded to the outcome data, revaluated the CT in every patient for the presence of epidural, subdural and/or subarachnoid hemorrhage, compression of the basal cisterns, and midline shift retrospectively.

2.4. Outcome Data. Cause of death and WLST data were collected from the medical records. For patients who received WLST, length of stay in ICU was noted. Functional outcome data, measured by the Glasgow Outcome Scale (GOS), were collected at three months (+one month) from records of outpatient clinic visits or correspondence from a neurological rehabilitation center. In case of missing data at three months, the first available GOS was used. If this was before three months' time, it was assessed with the threemonth follow-up data, since further deterioration was not expected. If follow-up data were only available after the fourmonth mark, they were separately analyzed. The GOS allows for objective assessment of the recovery of patients with brain damage in five categories [11]. Good functional outcome was defined as GOS $>3$.

2.5. Statistical Analysis. All statistical analyses were performed using IBM SPSS Statistics, version 21.0.0 (Armonk, NY, USA). Group differences between survivors and patients who died due to WLST were calculated using a MannWhitney $U$ test in case of continuous, nonnormally distributed, variables. In case of a different shape of distributions in each group, mean ranks were compared for analysis of significant differences between groups, and medians were only shown. Differences in distribution of categorical or ordinal variables between groups were calculated with the chi-square test of homogeneity. Fisher's exact test instead of a chi-square test was used if the expected cell count was less than five. Statistical significance was defined as $p<0.05$.

\section{Results}

3.1. Study Population. The search in the trauma registry generated a total of 367 patients with isolated moderate or severe TBI admitted between 2011 and 2015 to the ICU. After applying our exclusion criteria, 179 patients were included in this study (Figure 1). Of these patients, 55 (33\%) died during hospitalization (Table 1). The median age at time of the trauma was 57, the median AIS head was four, and the 


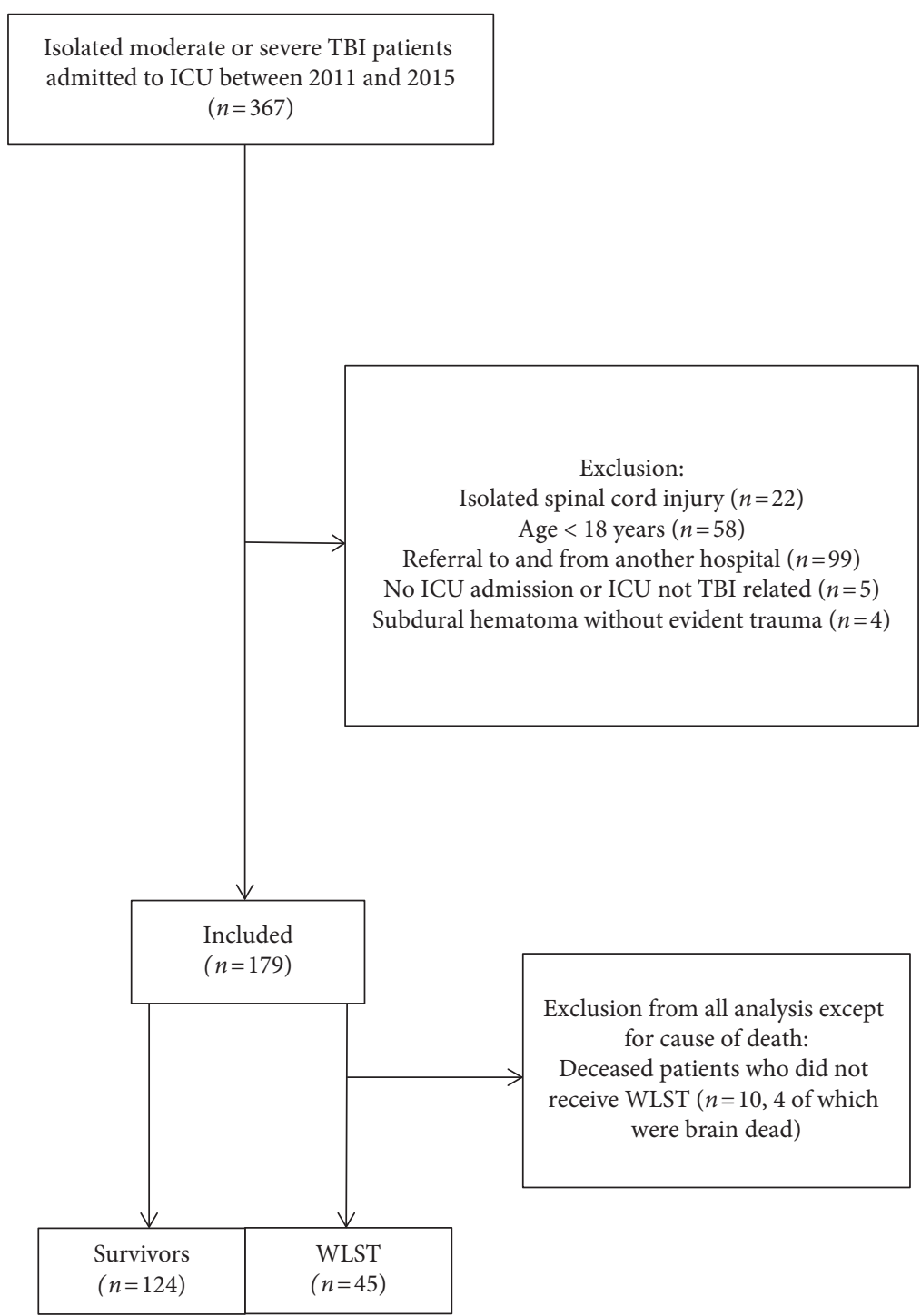

FIGURE 1: Flowchart of inclusion process. TBI: traumatic brain injury; WLST: withdrawal of life-sustaining treatment.

median ISS was 20 . Women accounted for $37 \%(n=62)$ of the patients (Table 1).

Patients for whom WLST was initiated were significantly older and had a median AIShead of five, whereas the AIShead of the non-WLST patients was four $(p<0.001)$. Use of coumarins, NOACs, and LMWH was more frequent in WLST patients $(p=0.043)$. The difference in mean rank of the Charlson Comorbidity Index was not statistically significant. GCS scores in ED were higher amongst those who did not receive WLST $(p=0.030)$. The absence of brainstem reflexes was more common in WLST patients (both $p<0.01$, Table 1). Furthermore, WLST patients were more often sedated before completion of the primary survey than non-WLST patients $(p=0.005)$. Subdural hemorrhage, compression of the basal cisterns, and midline shift on the initial CT head were more common in the group of patients who had WLST (all $p<0.05$, Table 1$)$.
3.2. Mortality, Surgical Intervention, Complications, and Neurological Outcome. Forty five patients (82\%) died following the decision to withdraw life-sustaining treatment. The decision to withdraw life-sustaining treatment was made on the day of admission in 18 cases $(40 \%)$. In $33 \%(n=6)$ of those patients, this decision was made whilst the patient was in ED.

$10(22 \%)$ of the WLST patients and $13(10 \%)$ of the nonWLST patients received an ICP meter $(p=0.049)$. The amount of patients who received neurosurgical decompression during admission did not differ between the non-WLST and WLST group ( $p=0.912$ ) (Table 2).

Median length of stay in ICU before the decision to withdraw life-sustaining treatment was made for patients who received WLST after the first day was 4 days. Of these 27 patients, 12 (44\%) suffered from a systemic complication at some point during their admission. In 50\% $(n=6)$ of these patients, this complication was solely pneumonia (Table 3 ). 
TABLE 1: Baseline characteristics and mortality.

\begin{tabular}{|c|c|c|c|c|}
\hline Variable & All patients $(n=169)$ & WLST $(n=45)$ & Non-WLST $(n=124)$ & $P$ value \\
\hline Mortality, $n(\%)^{*}$ & $55(33)$ & $100(45)$ & 0 & \\
\hline Median age in years (IQR) & $57(32.5)$ & $67(22)$ & $54(35.25)$ & \\
\hline Mean rank age & $\mathrm{n} / \mathrm{a}$ & 114.56 & 74.27 & $<0.001$ \\
\hline Median ISS (IQR) & $20(9)$ & $25(9)$ & $20(9)$ & 0.01 \\
\hline Median AIShead (IQR) & $4(1)$ & $5(1)$ & $4(1)$ & \\
\hline Mean rank AIShead & $\mathrm{n} / \mathrm{a}$ & 108.47 & 76.48 & $<0.001$ \\
\hline Female, $n(\%)$ & $62(37)$ & $16(36)$ & $46(37)$ & 0.854 \\
\hline Trauma mechanism, $n(\%)$ & & & & 0.061 \\
\hline Fall stairs or height & $53(32)$ & $19(44)$ & $34(28)$ & \\
\hline Fall low height/collaps or nos & $15(24)$ & $8(19)$ & $16(13)$ & \\
\hline Traffic accident: two wheels & $60(36)$ & $11(26)$ & $49(41)$ & \\
\hline Traffic accident: car & $12(7)$ & $1(2)$ & $11(10)$ & \\
\hline Traffic accident: pedestrian & $4(2)$ & $1(2)$ & $3(3)$ & \\
\hline Hit by subject & $6(4)$ & $0(0)$ & $6(5)$ & \\
\hline Penetrating injury & $3(2)$ & $2(5)$ & $1(1)$ & \\
\hline Hanging & $2(1)$ & $1(2)$ & $1(1)$ & \\
\hline Missing & $5(3)$ & $2(4)$ & $3(2)$ & \\
\hline \multicolumn{5}{|l|}{ Charlson Comorbidity Index (IQR) } \\
\hline Median & & $0(1)$ & $0(1)$ & \\
\hline Mean rank & & 88.37 & 81.23 & 0.302 \\
\hline \multicolumn{5}{|l|}{ Anticoagulant users, $n(\%)$} \\
\hline None or platelet aggregation inhibitors & & $32(82)$ & $116(94)$ & 0.043 \\
\hline Coumarins/heparines/NOAC & & $7(18)$ & $7(6)$ & \\
\hline Missing & & $6(13)$ & $1(1)$ & \\
\hline \multicolumn{5}{|l|}{ GCS in ED (IQR) } \\
\hline Median & & $7(8)$ & $8.5(5)$ & \\
\hline Mean rank & & 47.54 & 64.51 & 0.030 \\
\hline Sedated, $n(\%)$ & & $20(44)$ & $28(23)$ & \\
\hline \multicolumn{5}{|l|}{ Motor score in ED (IQR) } \\
\hline Median & & $5(4)$ & $5(1)$ & 0.009 \\
\hline Missing, $n(\%)$ & & $20(44)$ & $28(23)$ & \\
\hline \multicolumn{5}{|l|}{ Pupil reflexes in ED, $n(\%)$} \\
\hline None or one eye & & $21(50)$ & $17(15)$ & $<0.001$ \\
\hline Both eyes & & $21(50)$ & $96(85)$ & \\
\hline Missing & & $3(7)$ & $11(9)$ & \\
\hline \multicolumn{5}{|l|}{ Corneal reflexes in ED, $n(\%)$} \\
\hline None or one eye & & $11(58)$ & $4(17)$ & 0.009 \\
\hline Both eyes & & $8(42)$ & $20(83)$ & \\
\hline Missing & & $26(58)$ & $100(81)$ & \\
\hline Sedation, $n(\%)$ & & $20(44)$ & $28(23)$ & 0.005 \\
\hline \multicolumn{5}{|l|}{ Signs on first CT scan, $n(\%)$} \\
\hline Epidural hemorrhage & & $17(38)$ & $42(34)$ & 0.716 \\
\hline Subdural hemorrhage & & $43(96)$ & $93(75)$ & 0.004 \\
\hline Subarachnoidal hemorrhage & & $39(87)$ & $91(74)$ & 0.097 \\
\hline Compression basal cisterns & & $34(76)$ & $52(42)$ & $<0.001$ \\
\hline Midline shift & & $25(56)$ & $33(27)$ & 0.001 \\
\hline
\end{tabular}

WLST: withdrawal of life-sustaining treatment; AIShead: Abbreviated Injury Score of the head region. ${ }^{*} 10$ patients who died due to other causes than WLST are included in this analysis.

TABLE 2: Neurosurgery and ICP meter.

\begin{tabular}{lccc}
\hline Neurosurgery variables & WLST, $n=45$ & Non-WLST, $n=124$ & $P$ value \\
\hline Received ICP meter, $n(\%)$ & $10(22)$ & $13(10)$ & 0.049 \\
Underwent neurosurgical decompression, $n(\%)$ & $17(38)$ & $48(39)$ & 0.912 \\
\hline
\end{tabular}

WLST: withdrawal of life-sustaining treatment; ICP: intracranial pressure.

In-hospital mortality as a result of complications occurred in six patients (11\%). In two patients, these complications were cardiovascular: These patients died due to a cardiac arrest. Two patients died due to respiratory insufficiency, and one due to a fever in combination with the TBI. One patient had a fever, complicated by respiratory insufficiency, anuria, and diarrhea. This patient had several comorbidities. Four patients (7\%) progressed to death by neurological criteria (Figure 1). 
TABLE 3: ICU parameters for patients who did not receive WLST on the first day.

\begin{tabular}{lcc}
\hline ICU variables & WLST, $n=27$ & Non-WLST, $n=124$ \\
\hline Median length of stay in ICU in days (IQR) & $4(5)$ & $3(5)$ \\
Median length of stay in hospital in days (IQR) & $\mathrm{n} / \mathrm{a}$ & $17.5(21.75)$ \\
WLST following systemic complications & $n=12(44 \%)$ & $\mathrm{n} / \mathrm{a}$ \\
\hline
\end{tabular}

WLST: withdrawal of life-sustaining treatment; $\mathrm{n} / \mathrm{a}$ : not applicable.

None of our patients were discharged to a hospice, since death was expected to follow relatively quickly after the decision to withdraw life-sustaining care.

$71 \%(n=78)$ of the patients with a three-month or later follow-up scored $\geq 4$ on the GOS at three months (Table 4). No patient left the hospital with an unresponsive wakefulness syndrome or suffered from UWS after three months. Median GCS on the day of discharge was 15 (IQR: 0). One patient died within three months of discharge. Data concerning GOS were missing in $25 \%$ of survivors (Table 4 ).

\section{Discussion}

We have performed a single-center retrospective analysis on mortality rates, causes of death, WLST, and neurological outcome in patients who were admitted to the ICU with isolated moderate or severe TBI. The mortality rate was $33 \%$, which is comparable to that found in other developed countries $(30-40 \%)[1,5,12,13]$. The vast majority of patients died after a decision to withdraw life-sustaining treatment.

There are only four studies that have published rates of WLST in this group of patients. In-hospital mortality rates amongst patients with moderate to severe TBI varied between $10.8 \%$ and $44.1 \%$, whilst the WLST rates ranged between $45.0 \%$ and $86.6 \%$ in these studies: likely due to geographical and cultural differences $[1,5,14,15]$. Verkade et al. [8] looked at WLST rates in a Dutch ICU. They found that WLST preceded death in $95 \%$ of patients who passed away due to irreversible catastrophic cerebral damage [8].

Our WLST rates are at the higher end of the spectrum, when compared to the aforementioned studies; however, they are in range with the earlier published Dutch data [8]. We hypothesize that this may be partly due to cultural differences such as a smaller role of religion in the decisionmaking [6, 7]. Furthermore, we speculate that people in the Netherlands find quality of life extremely important and therefore might feel that life with UWS has no quality.

Patient wishes were always taken into account. If medical practitioners believe there is no chance of a decent outcome, they will inform the family that medical treatment would be futile. There are no cases in our database where families have doubted or opposed this statement. Unfortunately, due to the retrospective nature of our study, we are not able to trace preexistent patient documents, which might have influenced the decision.

WLST can be appropriate after severe traumatic brain injury to prevent a patient from staying alive at the cost of being left in a state of disability that might be against his or her wishes. However, WLST should not deny patients their chance of a good recovery. Numerous studies have identified several factors with a predictive relationship with outcome after TBI. So far, no model has proven to be perfect, but two widely used prognostication models are the IMPACT score and the CRASH score [20-22]. The risk that WLST may lead to self-fulfilling prophecies, when the prognostic model confirms itself due to physicians basing the decision to WLST on the factors present in this model, has previously been acknowledged for patients with various types of acute brain injury [16-18].

In our study, the decision to withdraw life-sustaining treatment was made in the very acute stage of the disease. Our findings are similar to those of Turgeon et al. [5] where $45.6 \%$ of patients who died with WLST did so within the first three days. There is a possibility that patients might have shown clinical improvement if the decision to WLST would have been postponed. In some cases, the decision to WLST has been made when the patient was sedated. The neurological state of these patients has therefore not been assessed. We believe the decision to not discontinue sedation is based on the facts that some patients are clinically not well enough to discontinue sedation or their CT head shows unsalvageable brain damage.

The Neurocritical Care Society therefore suggests delaying withdrawal of treatment and treatment limitations for at least 72 hours in cases of devastating brain injury to give the patient the chance to recover and reduce the risk of prematurely forgoing treatments that could provide clinical benefit [19]. Even though these guidelines were not written for TBI specifically, this raises the concern that the decision to withdraw treatment was made too early in some of the patients in this study.

In addition, the amount of patients who received ICP monitoring was relatively low, when compared to other studies $[1,14]$. Even though we have not formally investigated this, we believe that, in line with hospital practice, patients who did not receive an ICP and/or neurosurgical operation were either considered to have a relatively minor injury or unsalvageable catastrophic cerebral damage.

This study has several limitations. Firstly, due to the retrospective nature of this study, we encountered several missing data. One example is the agent and dosage used to sedate the patient. Therefore, we were only able to tell whether the patient was sedated before completion of the primary survey and not if and how this could have affected prognosis. The most important of missing data is that the GOS was not available for all of our patients. Furthermore, at the three-month mark, many patients were still in rehabilitation clinics, but expected to be able to return to an independent life. As such, there is a broad range of neurological outcomes amongst those with a GOS of three, ranging from patients requiring a tracheostomy to those 
TABLE 4: GOS score and destination after discharge of the survivor group. The GOS score at three to four months or less of patients who were alive at discharge, expressed in percentage per GOS score.

\begin{tabular}{lcccc}
\hline $\begin{array}{l}\text { Glasgow } \\
\text { Outcome Scale }\end{array}$ & $\begin{array}{c}\text { Patients assessed at three } \\
\text { months, } n=124(\%)\end{array}$ & $\begin{array}{c}\text { Patients assessed after three } \\
\text { months, } n=23(\%)\end{array}$ & $\begin{array}{c}\text { All assessed patients, } \\
n=124(\%)\end{array}$ & $\begin{array}{c}\text { Discharged to } \\
n=124(\%)\end{array}$ \\
\hline 1 & $1(1)$ & $0(0)$ & $1(0)$ & $50(40)$ \\
2 & $0(0)$ & $0(0)$ & $0(0)$ & Home \\
Rehab & Home against \\
3 & $31(31)$ & $0(0)$ & $31(28)$ & medical advice \\
4 & $52(51)$ & $5(56)$ & $57(52)$ & \\
5 & $17(17)$ & $4(44)$ & $21(19)$ & Psychiatry ward \\
Missing & $23(19)$ & $14(61)$ & $14(11)$ & $2(2)$ \\
\hline
\end{tabular}

The individual values are rounded to the nearest percent and may not total $100 \%$. Score 1 is defined as death and score 2 is unresponsive wakefulness syndrome. Score 3 is defined as severe injury with permanent need for help with daily living, and score 4 is moderate disability; no need for assistance in everyday life, employment is possible, but may require special equipment. When a patient scores a 5 , he or she has only minor deficits in the physical, social, or psychological domain [16].

who are on the verge of discharge from the rehabilitation clinic. Patients are not likely to have made their full recovery yet at three months; however, most follow-up data were available until three months. Therefore, future research including a longer follow-up period of these patients is necessary to determine the definite neurological outcome. Using the eight-point GOS scale (the extended GOS) can also specify functional outcome even more and has been recommended in the literature [23]. Unfortunately, a retrospective analysis makes filling out the eight-point scale too difficult; therefore, the five-point scale was considered the more appropriate option, hoping this would prevent misclassification and limit missing data. Furthermore, a comparison of WLST rates between several trauma centers is warranted to establish the exact influence of WLST on mortality and outcome data. In addition, as of today, there is no standard protocol regarding WLST decisions. The decision to withdraw treatment is always taken by the treating physicians including trauma surgeon, neurosurgeon/neurologist, and intensivist and needs to be unanimous before treatment is withdrawn. The lack of standardized documentation of the considerations leading to this decision and therefore lack of analyzed data regarding this subject is a limitation of this study. Finally, we would like to propose a study that investigates the process of, and influences on, the decision to withdraw care.

\section{Conclusion}

The vast majority of in-hospital deaths after moderate or severe TBI occur following a decision to withdraw lifesustaining treatments. Functional outcome of TBI survivors is generally good.

\section{Data Availability}

The data from the medical records used to support the findings of this study are restricted by the Medisch Ethische Toetsingscommissie (Medical Ethical Committee) in order to protect patient privacy. Pseudonymized data are available from the corresponding author for researchers who meet the criteria for access to confidential data.

\section{Conflicts of Interest}

The authors declare that they have no conflicts of interest.

\section{References}

[1] S. Izzy, R. Compton, R. Carandang, W. Hall, and S. Muehlschlegel, "Self-fulfilling prophecies through withdrawal of care: do they exist in traumatic brain injury, too?," Neurocritical Care, vol. 19, no. 3, pp. 347-363, 2013.

[2] C. A. Taylor, J. M. Bell, M. J. Breiding, and L. Xu, "Traumatic brain injury-related emergency department visits, hospitalizations, and deaths - United States, 2007 and 2013," MMWR. Surveillance Summaries, vol. 66, no. 9, pp. 1-16, 2017.

[3] M. Majdan, D. Plancikova, A. Maas et al., "Years of life lost due to traumatic brain injury in Europe: a cross-sectional analysis of 16 countries," PLoS Medicine, vol. 14, no. 7, article e1002331, 2017.

[4] A. C. Gunning, K. W. W. Lansink, K. J. P. van Wessem et al., "Demographic patterns and outcomes of patients in level I trauma centers in three international trauma systems," World Journal of Surgery, vol. 39, no. 11, pp. 2677-2684, 2015.

[5] A. F. Turgeon, F. Lauzier, J. F. Simard et al., "Mortality associated with withdrawal of life-sustaining therapy for patients with severe traumatic brain injury: a Canadian multicentre cohort study," Canadian Medical Association Journal, vol. 183, no. 14, pp. 1581-1588, 2011.

[6] M. Geurts, M. R. Macleod, G. J. M. W. van Thiel, J. van Gijn, L. J. Kappelle, and H. B van Der Worp, "End-of-life decisions in patients with severe acute brain injury," The Lancet Neurology, vol. 13, no. 5, pp. 515-524, 2014.

[7] C. L. Sprung, S. L. Cohen, P. Sjokvist et al., "End-of-life practices in European intensive care units: the Ethicus study," JAMA, vol. 290, no. 6, pp. 790-797, 2003.

[8] M. A. Verkade, J. L. Epker, M. D. Nieuwenhoff, J. Bakker, and E. J. Kompanje, "Withdrawal of life-sustaining treatment in a mixed intensive care unit: most common in patients with catastrophic brain injury," Neurocritical Care, vol. 16, no. 1, pp. 130-135, 2012.

[9] P. Fraccaro, E. Kontopantelis, M. Sperrin et al., "Predicting mortality from change-over-time in the Charlson Comorbidity Index: a retrospective cohort study in a data-intensive UK health system," Medicine, vol. 95, no. 43, article e4973, 2016.

[10] M. Yurkovich, J. A. Avina-Zubieta, J. Thomas, M. Gorenchtein, and D. Lacaille, "A systematic review identifies valid comorbidity 
indices derived from administrative health data," Journal of Clinical Epidemiology, vol. 68, no. 1, pp. 3-14, 2015.

[11] J. T. Lindsay Wilson, L. E. Pettigrew, and G. M. Teasdale, "Structured interviews for the Glasgow Outcome Scale and the extended Glasgow Outcome Scale: guidelines for their use," Journal of Neurotrauma, vol. 15, no. 8, pp. 573-585, 1998.

[12] A. Marmarou, J. Lu, I. Butcher et al., "IMPACT database of traumatic brain injury: design and description," Journal of Neurotrauma, vol. 24, no. 2, pp. 239-250, 2007.

[13] E. J. Lilley, J. W. Scott, J. S. Weissman et al., "End-of-Life Care in Older Patients After Serious or Severe Traumatic Brain Injury in Low-Mortality Hospitals Compared With All Other Hospitals," JAMA Surgery, vol. 153, no. 1, p. 44, 2017.

[14] A. Robertsen, R. Førde, N. O. Skaga, and E. Helseth, "Treatment-limiting decisions in patients with severe traumatic brain injury in a Norwegian regional trauma center," Scandinavian Journal of Trauma, Resuscitation and Emergency Medicine, vol. 25, no. 1, p. 44, 2017.

[15] M. J. Sise, C. B. Sise, J. F. Thorndike, J. E. Kahl, R. Y. Calvo, and S. R. Shackford, "Withdrawal of care: a 10-year perspective at a Level I trauma center," Journal of Trauma and Acute Care Surgery, vol. 72, no. 5, pp. 1186-1193, 2012.

[16] K. J. Becker, A. B. Baxter, W. A. Cohen et al., "Withdrawal of support in intracerebral hemorrhage may lead to self-fulfilling prophecies," Neurology, vol. 56, no. 6, pp. 766-772, 2001.

[17] R. G. Geocadin, M. A. Peberdy, and R. M. Lazar, "Poor survival after cardiac arrest resuscitation: a self-fulfilling prophecy or biologic destiny?," Critical Care Medicine, vol. 40, no. 3, pp. 979-980, 2012.

[18] M. A. Kirkman, T. Jenks, O. Bouamra, A. Edwards, D. Yates, and M. H. Wilson, "Increased mortality associated with cerebral contusions following trauma in the elderly: bad patients or bad management?" Journal of Neurotrauma, vol. 30, no. 16, pp. 1385-1390, 2013.

[19] M. J. Souter, P. A. Blissitt, S. Blosser et al., "Recommendations for the critical care management of devastating brain injury: prognostication, psychosocial, and ethical management: a position statement for healthcare professionals from the neurocritical care society," Neurocritical Care, vol. 23, no. 1, pp. 4-13, 2015.

[20] B. Roozenbeek, H. F. Lingsma, F. E. Lecky et al., "Prediction of outcome after moderate and severe traumatic brain injury: external validation of the International Mission on Prognosis and Analysis of Clinical Trials (IMPACT) and Corticoid Randomisation After Significant Head injury (CRASH) prognostic models," Critical Care Medicine, vol. 40, no. 5, pp. 1609-1617, 2012.

[21] M. Mjadan, H. F. Lingsma, D. Nieboer, W. Mauritz, M. Rusnak, and E. W. Steyerberg, "Performance of IMPACT, CRASH and Nijmegen models in predicting six month outcome of patients with severe or moderate TBI: an external validation study," Scandinavian Journal of Trauma, Resuscitation and Emergency Medicine, vol. 22, no. 1, p. 68, 2014.

[22] E. W. Steyerberg, N. Mushkudiani, P. Perel et al., "Predicting outcome after traumatic brain injury: development and international validation of prognostic scores based on admission characteristics," PLoS Medicine, vol. 5, no. 8, article e165, 2008.

[23] E. A. Wilde, G. G. Whitneck, J. Bogner et al., "Recommendations for the use of common outcome measures in traumatic brain injury research," Archives of Physical Medicine and Rehabilitation, vol. 91, pp. 1650-1660, 2010. 


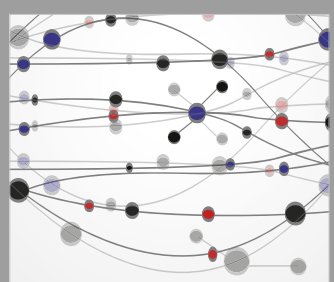

The Scientific World Journal
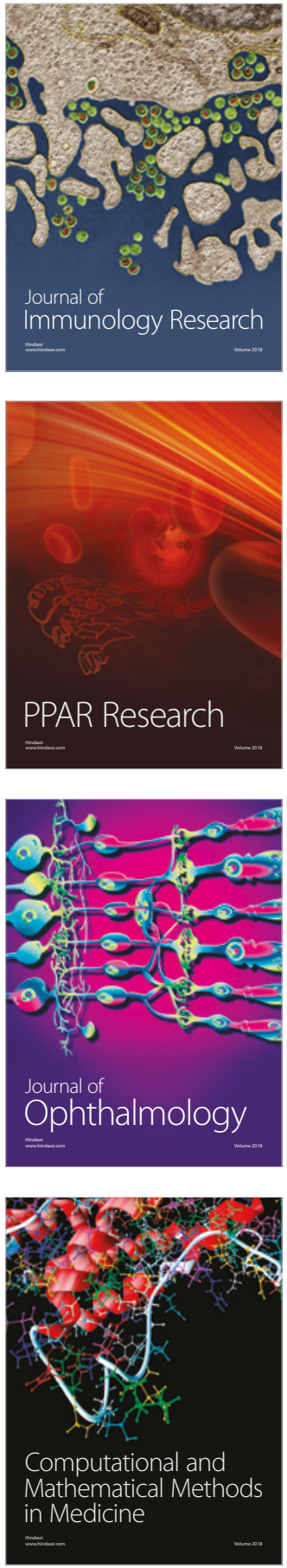

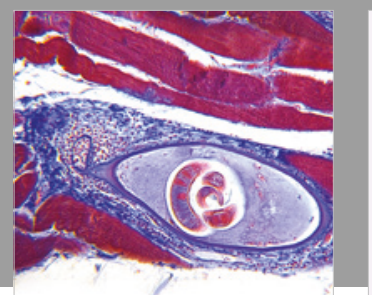

Gastroenterology Research and Practice

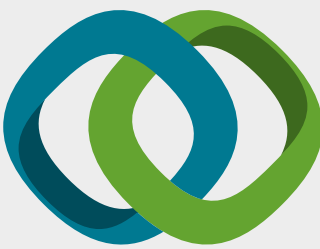

\section{Hindawi}

Submit your manuscripts at

www.hindawi.com
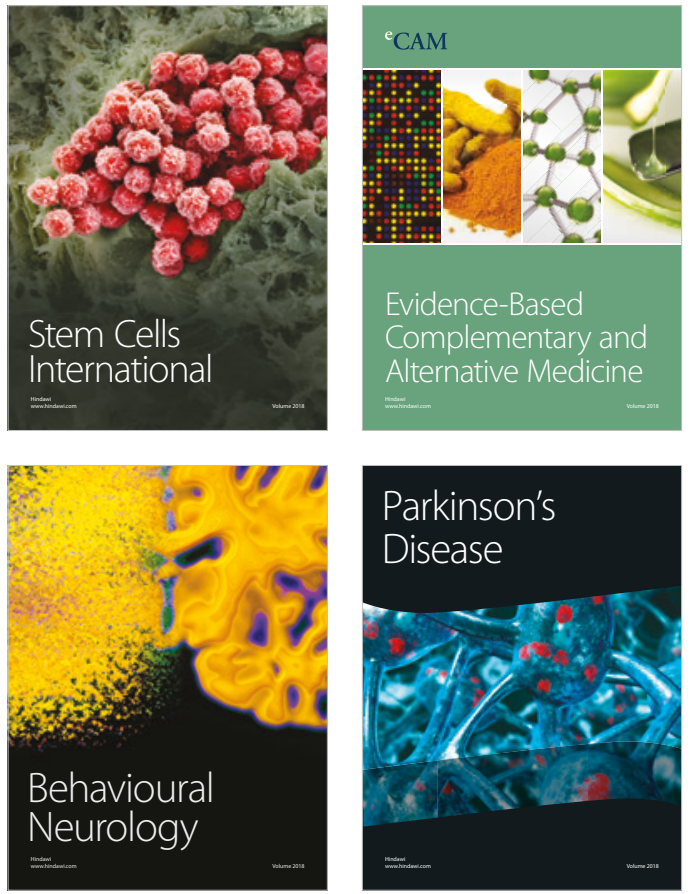

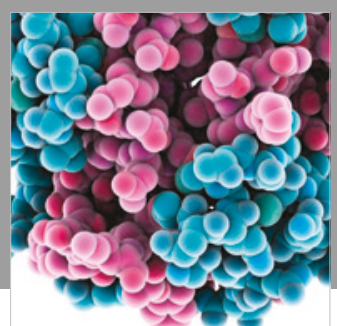

ournal of

Diabetes Research

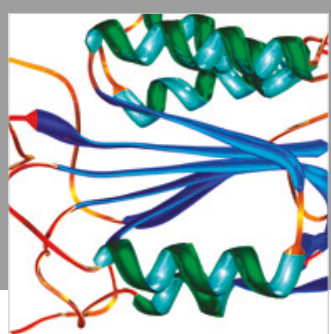

Disease Markers
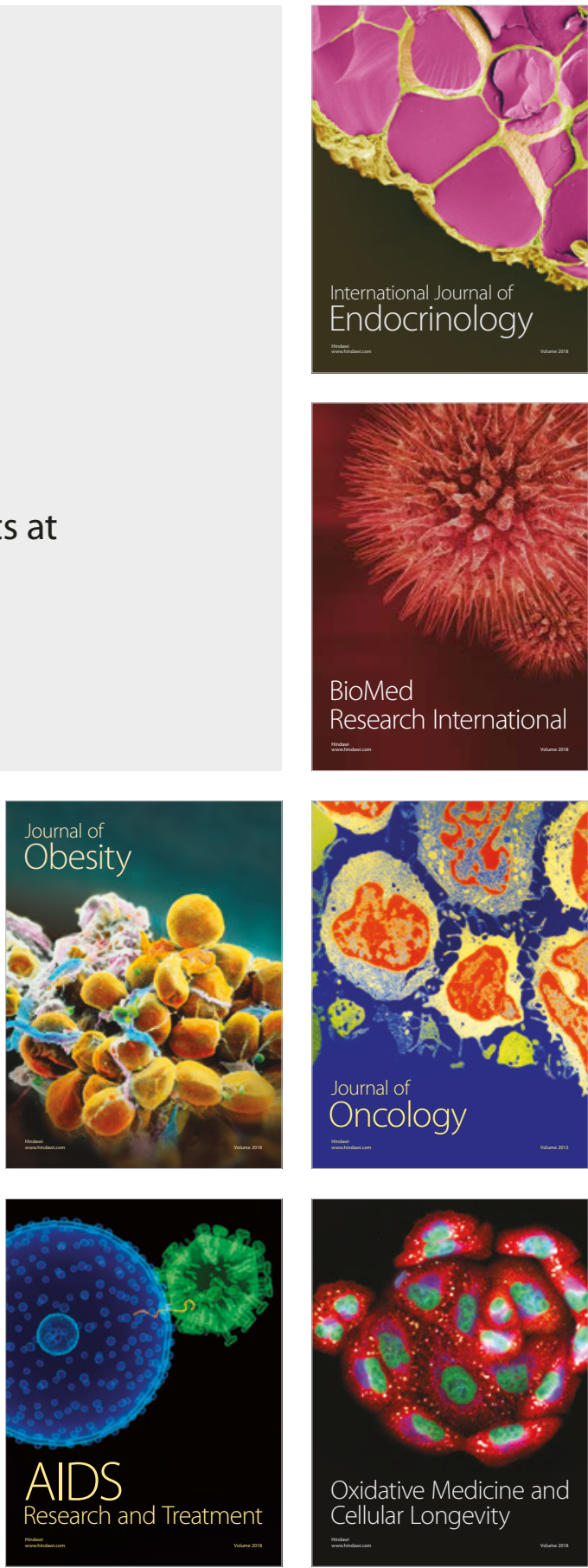